\title{
Downregulated MFAP4 expression is correlated with macrophage polarization and indicates poor prognosis in lung adenocarcinoma
}

\section{Zhiyan Luo}

Shenzhen Hospital of Integrated Traditional Chinese and Western Medicine

\section{Weifeng Yu}

The Second Affiliated Hospital of Guangzhou University of Chinese Medicine

\section{Tiegang Yue}

Shenzhen Hospital of Integrated Traditional Chinese and Western Medicine

\section{Yinghan Long}

Shenzhen Hospital of Integrated Traditional Chinese and Western Medicine

\section{Yuguang Chen}

Shenzhen Hospital of Integrated Traditional Chinese and Western Medicine

\section{Yong Jiang ( $\nabla$ jiangyongsz@163.com )}

Shenzhen Hospital of Integrated Traditional Chinese and Western Medicine

\section{Research Article}

Keywords: MFAP4, LUAD, immune cell, TNM stage, gender, age, pathologic stage

Posted Date: October 25th, 2021

DOl: https://doi.org/10.21203/rs.3.rs-959751/v1

License: (c) (i) This work is licensed under a Creative Commons Attribution 4.0 International License. Read Full License 


\section{Abstract}

Microfiber-associated protein 4(MFAP4) plays a crucial role in several types of cancers; however, its role in lung adenocarcinoma(LUAD) remains elusive. Here, we investigated MFAP4 expression in LUAD tissues at the mRNA and protein levels respectively. Kaplan-Meier survival analysis was performed to investigate the prognostic role of MFAP4 in LUAD. Cox regression analysis was performed to explore the correlation between the clinical characteristics and MFAP4 expression. Moreover, the confidence level between MFAP4 expression and immune cell infiltration in LUAD tissues was calculated using TIMER and validated using GEPIA. MFAP4 expression was downregulated in LUAD at the gene and protein levels $(\mathrm{P}<$ 0.001). MFAP4 expression might be an independent risk factor for overall survival $(P=0.023)$. Moreover, the $\mathrm{C}$-index of the nomogram model was 0.664 , implying a good predictive capability of this model. Additionally, low MFAP4 expression significantly correlated with immune-related gene expression; further analysis showed that downregulated MFAP4 expression was positively correlated with M2 macrophage infiltration and negatively correlated with M1 macrophage infiltration. Our study indicated that MFAP4 expression could be a potential prognostic biomarker for LUAD. Low-MFAP4 expression indicates poor prognosis for LUAD patients $(P=0.011)$, and the underlying mechanism may be related to the polarization of the macrophages into the $\mathrm{M} 2$ phenotype.

\section{Introduction}

Lung cancer is the most commonly diagnosed cancer and has the highest mortality rate (approximately $18.4 \%$ ) among all cancers, accounting for more than 2 million deaths globally, in $2018^{1}$. Lung adenocarcinoma (LUAD) accounts for $40 \%$ of lung cancer diagnoses and is its most prevalent subtype ${ }^{2}$. Currently, the management of LUAD involves surgical removal during the early stages and chemoradiotherapy during the advanced stages; however, the treatment outcomes are not satisfactory. The clinical implementation of the precision medicine strategy, in 2001, commenced a new era in cancer treatment ${ }^{3}$, demonstrating an exemplary, increased median overall survival ${ }^{4}$. However, the effects of drug resistance ${ }^{5}$ and the possibility of unidentified therapeutic targets ${ }^{6}$ cannot be ignored. Thus, there is an urgent need to identify novel prognostic biomarkers to aid clinical decision making and therapies to improve outcome.

Microfiber-associated protein 4 (MFAP4) is a glycoprotein that is present in the extracellular matrix (ECM), affecting its functions, such as tissue homeostasis, microfibril assembly, and elastinogenesis ${ }^{7}$. At present, the studies on MFAP4 function have primarily focused on the non-invasive assessment for common chronic disease, including liver fibrosis ${ }^{8}$, chronic obstructive pulmonary disease ${ }^{9}$ and cardiovascular disease ${ }^{10}$. As matricellular proteins belonging to the fibrinogen-related protein superfamily, MFAP2 and MFAP5 have been reportedly associated with many tumor types ${ }^{11,12}$. Therefore, these findings suggest that MFAP4 may potentially be a novel cancer prognostic biomarker. Unfortunately, only few reports have described the role of MFAP4 in LUAD. 
Here, we explored the prognostic value of MFAP4 in LUAD using the survival data from The Cancer Genome Atlas (TCGA), Genotype-Tissue Expression(GTEx), and Gene Expression Omnibus(GEO) database. Moreover, considering the important role of immune cells in the tumor microenvironment (TME), we also performed immune infiltration analysis to assess the relationship between MFAP4 expression and immune infiltration.

\section{Results}

\section{Down-regulated MFAP4 expression in LUAD}

First, we analyzed the expression of MFAP4 at the pan-cancer level using the TIMER database. Box plots were used to display the distributions of gene expression levels. MFAP4 expression was downregulated in 16 types of cancers (Figure 1a). In order to analyze the differential expression of MFAP4 in LUAD, we compared the normal and tumor tissues from TCGA database between the paired and unpaired conditions, and the results showed that MFAP4 expression was lower in the LUAD tissues (Figure 1b-c). Moreover, we achieved similar results after adding the data from an unpaired, normal tissue from the GTEx database (Figure 1d). Furthermore, we also obtained MFAP4 protein-level data from the Human Protein Atlas (HPA) database, the results of immunohistochemistry indicated that the MFAP4 levels in tumors(Figure 2b) were not higher than those in normal tissues(Figure 2a).

Figure 1.Down-regulated expression of MFAP4 in Pan-cancer and LUAD.(a)MAFP4 expression in pancancer data from TCGA. (b-c)MFAP4 expression in normal and tumor tissues in LUAD from TCGA database. (d)MFAP4 expression in normal and tumor tissues in LUAD from TCGA and GTEx database. ${ }^{*} p<0.05, * \star p<0.01, * * * p<0.001$

\section{Baseline clinical characteristics of LUAD patients}

The clinicopathological characteristics assessed included TNM stage, gender, age, pathologic stage, primary therapy outcome, residual tumor, smoker, and anatomic neoplasm subdivision (Table 1). The proportion of women and men in this study was similarly equal to (50\%); however, but the disease is slightly more common in women. From the perspective of personal life habits, smokers accounts for the vast majority of proportion the patients compared with non-smokers. Moreover, from the perspective of pathologic stage, more than half of the patients (55.7\%) were in the initial stages of LUAD. 
Table 1

Clinical characteristics of LUAD patients based on TCGA

\begin{tabular}{|c|c|c|c|}
\hline Clinical Characteristics & & $\mathbf{N}$ & $\%$ \\
\hline \multirow[t]{4}{*}{ T stage } & $\mathrm{T} 1$ & 107 & 32.9 \\
\hline & $\mathrm{T} 2$ & 189 & 54.3 \\
\hline & T3 & 49 & 9.3 \\
\hline & $\mathrm{T} 4$ & 19 & 3.5 \\
\hline \multirow[t]{4}{*}{ N stage } & No & 348 & 67.1 \\
\hline & $\mathrm{N} 1$ & 95 & 18.3 \\
\hline & $\mathrm{N} 2$ & 74 & 14.2 \\
\hline & N3 & 2 & 0.4 \\
\hline \multirow[t]{2}{*}{ M stage } & MO & 361 & 93.6 \\
\hline & M1 & 25 & 6.4 \\
\hline \multirow[t]{4}{*}{ Pathologic stage } & Stage I & 294 & 55.7 \\
\hline & Stage II & 123 & 23.4 \\
\hline & Stage III & 84 & 16 \\
\hline & Stage IV & 26 & 4.9 \\
\hline \multirow[t]{4}{*}{ Primary therapy outcome } & PD & 71 & 16 \\
\hline & SD & 37 & 8.3 \\
\hline & PR & 6 & 1.3 \\
\hline & $\mathrm{CR}$ & 332 & 74.4 \\
\hline \multirow[t]{2}{*}{ Gender } & Female & 286 & 53.5 \\
\hline & Male & 249 & 46.5 \\
\hline \multirow[t]{2}{*}{ Age (years) } & $<=65$ & 255 & 49.5 \\
\hline & $>65$ & 261 & 50.5 \\
\hline \multirow[t]{3}{*}{ Residual tumor } & Ro & 355 & 95.4 \\
\hline & $\mathrm{R} 1$ & 13 & 3.5 \\
\hline & $\mathrm{R} 2$ & 4 & 1.1 \\
\hline Smoker & No & 75 & 14.4 \\
\hline
\end{tabular}




\section{\begin{tabular}{|llll|}
\hline Clinical Characteristics & & N & $\%$ \\
\hline & Yes & 446 & 85.6 \\
\hline Anatomic neoplasm subdivision & Left & 205 & 39.4 \\
& Right & 315 & 60.6 \\
\hline Anatomic neoplasm subdivision2 & Central Lung & 62 & 32.8 \\
\hline & Peripheral Lung & 127 & 67.2 \\
\hline
\end{tabular} \\ Low MFAP4 expression was associated poor prognosis in LUAD patients}

In order to clarify the role of MFAP4 expression as a prognostic biomarker for LUAD, LUAD patients survival data from TCGA were divided into two groups (high- and low-MFAP4 expression) based on the median value of MFAP4 expression. Kaplan-Meier survival analysis indicated that low-MFAP4 expression correlated with poor prognosis, whereas high-MFAP4 expression represented improved survival ( $p=0.011$, Figure 3a). Subsequently, we performed a series subgroup analyses to examine the correlations of MFAP4 expression with clinical features. Undoubtedly, low-MFAP4 expression positively correlated with poor prognosis in subgroups such as T1 and T2 stage, N0 and N1 stage, N2 stage, M0 stage, R0 stage of residual tumor, more than 65 years old, female, smoker, tumor occurred in the left lung, and the response on primary therapy was PD and SD (Figure 3b-3k). To better understand the association between MFAP4 expression and overall survival of LUAD patients, we also analyzed the LUAD patient survival data from GSE13213 and GSE31210 using the PrognoScan database.

Downregulated expression of MFAP4 indicated poor survival in these two datasets(Figure 4), similar to the results mentioned earlier. Collectively, these results indicated that poor LUAD patient prognosis was associated with downregulated MFAP4 expression.

Furthermore, univariate and multivariate Cox regression analyses were performed to assess the association between survival and clinical characteristics. In addition to $\mathrm{T}$ stage, $\mathrm{N}$ stage, and $\mathrm{M}$ stage, which were unique to univariate analysis, multivariate analysis results indicated that pathologic stage, primary therapy outcome, residual tumor, and MFAP4 expression were the independent prognostic risk factors for LUAD patients (Table 2). Therefore, MFAP4 may be a potential prognostic biomarker for LUAD. Moreover, the results of the univariate analysis were visualized using a forest plot to display the hazard ratios of clinical features (Figure 5a). A nomogram was constructed to predict the OS at 1, 3, and 5 years based on the results of multivariate analysis (Figure $5 b$ ). Moreover, a calibration plot was drawn to validate the prediction model (Figure 5c), the C-index of this model was 0.664 , which implied a good agreement between the nomogram model results and actual outcomes.

\section{Table2. Univeriate and multivariate Cox analysis of clinical characteristics associated with OS}




\begin{tabular}{|c|c|c|c|c|}
\hline \multirow[t]{2}{*}{ Characteristics } & \multicolumn{2}{|c|}{ Univariate analysis } & \multicolumn{2}{|c|}{ Multivariate analysis } \\
\hline & $\mathrm{HR}(95 \% \mathrm{Cl})$ & $\begin{array}{l}P \\
\text { value }\end{array}$ & $\begin{array}{l}\mathrm{HR}(95 \% \\
\mathrm{Cl})\end{array}$ & $\begin{array}{l}\mathrm{P} \\
\text { value }\end{array}$ \\
\hline T stage (T2\&T3\&T4 vs. T1) & $\begin{array}{l}1.728 \\
(1.229- \\
2.431)\end{array}$ & 0.002 & $\begin{array}{l}1.115 \\
(0.638- \\
1.949)\end{array}$ & 0.703 \\
\hline N stage (N1\&N2\&N3 vs. N0) & $\begin{array}{l}2.601 \\
(1.944- \\
3.480)\end{array}$ & $<0.001$ & $\begin{array}{l}1.493 \\
(0.925- \\
2.411)\end{array}$ & 0.101 \\
\hline M stage (M1 vs. M0) & $\begin{array}{l}2.136 \\
(1.248- \\
3.653)\end{array}$ & 0.006 & $\begin{array}{l}1.108 \\
(0.425- \\
2.889)\end{array}$ & 0.834 \\
\hline $\begin{array}{l}\text { Pathologic stage (Stage III\&Stage IV vs. Stage } \\
\text { I\&Stage II) }\end{array}$ & $\begin{array}{l}2.664 \\
(1.960- \\
3.621)\end{array}$ & $<0.001$ & $\begin{array}{l}1.978 \\
(1.091- \\
3.586)\end{array}$ & 0.025 \\
\hline Primary therapy outcome (PR\&CR vs. PD\&SD) & $\begin{array}{l}0.377 \\
(0.268- \\
0.530)\end{array}$ & $<0.001$ & $\begin{array}{l}0.316 \\
(0.190- \\
0.524)\end{array}$ & $<0.001$ \\
\hline Gender (Male vs. Female) & $\begin{array}{l}1.070 \\
(0.803- \\
1.426)\end{array}$ & 0.642 & & \\
\hline Age (>65 vs. <=65) & $\begin{array}{l}1.223 \\
(0.916- \\
1.635)\end{array}$ & 0.172 & & \\
\hline Residual tumor (R1\&R2 vs. R0) & $\begin{array}{l}3.879 \\
(2.169- \\
6.936)\end{array}$ & $<0.001$ & $\begin{array}{l}4.907 \\
(2.023- \\
11.906)\end{array}$ & $<0.001$ \\
\hline $\begin{array}{l}\text { Anatomic neoplasm subdivision (Right vs. } \\
\text { Left) }\end{array}$ & $\begin{array}{l}1.037 \\
(0.770- \\
1.397)\end{array}$ & 0.810 & & \\
\hline $\begin{array}{l}\text { Anatomic neoplasm subdivision2 (Peripheral } \\
\text { Lung vs. Central Lung) }\end{array}$ & $\begin{array}{l}0.913 \\
(0.570- \\
1.463)\end{array}$ & 0.706 & & \\
\hline Smoker (Yes vs. No) & $\begin{array}{l}0.894 \\
(0.592- \\
1.348)\end{array}$ & 0.591 & & \\
\hline MFAP4 (High vs. Low) & $\begin{array}{l}0.688 \\
(0.515- \\
0.919)\end{array}$ & 0.011 & $\begin{array}{l}0.558 \\
(0.336- \\
0.924)\end{array}$ & 0.023 \\
\hline
\end{tabular}

\section{The association between MFAP4 expression and immune infiltration}


The TME considerably influences the prognosis. As the above findings showed that MFAP4 might be a prognostic biomarker for LUAD, it is important to further explore the association between MFAP4 expression and immune cell infiltration. Thus, we calculated the confidence level between MFAP4 expression and immune infiltration to explore their correlation using TIMER. As the figure shows, there was a significant correlation between MFAP4 expression and six tumor-infiltrating immune cell (TIIC) subsets, namely, B cell, CD8+ T cell, CD4+ T cell, macrophage, monocyte, neutrophil, and dendritic cell; moreover, MFAP4 expression was negatively correlated with tumor purity (Figure 6a). Furthermore, we found that the infiltration of $B$ cell $(P<0.001)$ and dendritic cell $(P=0.048)$ and MFAP4 expression $(P=$ 0.014 ) were correlated with LUAD prognosis(Figure $6 \mathrm{~b}$ ). Because the macrophage infiltration level demonstrated the strongest significant correlation with MFAP4, we investigated whether MFAP4 expression influences macrophage polarization. To this end, we examined the association between MFAP4 expression and M1/M2 macrophage. Interestingly, MFAP4 expression was positively correlated with M2 macrophages and negatively correlated with M1 macrophages (Figure $6 c-6 d$ ). These results showed that MFAP4 expression was correlated with immune cell infiltrations in LUAD tissues and most likely influenced the polarization of macrophages.

Figure 6. MFAP4 expression is correlated with immune cell infiltrations in LUAD.(a)MFAP4 expression is associated with six tumor-infiltrating immune cells subsets. (b)Kaplan-Meier plots of MFAP4 expression and the infiltration level of six tumor-infiltrating immune cells subsets. (c)MFAP4 expression and infiltration level of M1/M2 macrophages before purity adjustment. (d) MFAP4 expression and infiltration level of M1/M2 macrophages after purity adjustment.

\section{Relationship between MFAP4 expression and immune markers in LUAD patients}

Subsequently, we evaluated the correlations between MFAP4 expression and immune-related markers using TIMER. The gene sets comprised marker genes of different immune cell subsets, including macrophages [M1/M2 and tumor-associated macrophages (TAMs)], monocytes, NK cells, dendritic cells, neutrophils, as well as B and T cell subsets (Tfh, Th1, Th2, Th9, Th17, Th22, Treg, and T cell exhaustion). The number of correlated gene markers increased after adjusting for tumor purity (Table 3). Considering the fact that the macrophage infiltration level presented the most significant correlation, we analyzed and visualized the correlation of MFAP4 expression and macrophage markers in TIMER (Figure 7); moreover, we validated the correlation in GEPIA (Table 4). As expected, the analysis of the two databases led to the same result that MFAP4 expression correlated with macrophage infiltration. 
Table 3

Correlation analysis between MFAP4 and markers of immune cells in TIMER

\begin{tabular}{|c|c|c|c|c|c|}
\hline \multirow[t]{2}{*}{ Cell type } & \multirow[t]{2}{*}{ Gene marker } & \multicolumn{2}{|l|}{ None } & \multicolumn{2}{|l|}{ Purity } \\
\hline & & Cor & $\mathrm{p}$ & Cor & $p$ \\
\hline \multirow[t]{3}{*}{ B cell } & CD19 & 0.369 & $\star \star \star *$ & -0.232 & $\star \star \star$ \\
\hline & KRT20 & 0.106 & $\star *$ & 0.089 & * \\
\hline & CD38 & 0.19 & $\star \star \star$ & 0.052 & 0.248 \\
\hline \multirow[t]{2}{*}{ CD8+T cell } & CD8A & 0.172 & $\star \star \star *$ & 0.007 & 0.88 \\
\hline & CD8B & 0.106 & ** & -0.024 & 0.59 \\
\hline \multirow[t]{3}{*}{ Tfh } & CXCR5 & 0.431 & 1 & 0.303 & $\star \star \star$ \\
\hline & ICOS & 0.306 & $\star \star \star$ & 0.132 & * \\
\hline & BCL-6 & 0.225 & $\star \star \star *$ & 0.233 & $\star \star \star *$ \\
\hline \multirow[t]{7}{*}{ Th1 } & IL12RB2 & -0.064 & 0.144 & -0.168 & $\star \star \star *$ \\
\hline & IL27RA & 0.232 & $\star \star \star \star$ & 0.149 & $\star \star \star *$ \\
\hline & STAT4 & 0.344 & $\star \star \star *$ & 0.211 & $\star \star \star *$ \\
\hline & IFNG & -0.091 & * & -0.256 & $\star \star \star *$ \\
\hline & TBX21 & 0.258 & $\star \star \star *$ & 0.105 & * \\
\hline & STAT1 & 0.031 & 0.768 & -0.123 & $\star \star *$ \\
\hline & TNF- $a$ & 0.247 & $\star \star \star *$ & 0.116 & $\star \star$ \\
\hline \multirow[t]{4}{*}{ Th2 } & CCR3 & 0.2 & $\star \star \star *$ & 0.111 & * \\
\hline & STAT6 & 0.244 & $\star \star \star *$ & 0.283 & $\star \star \star *$ \\
\hline & GATA3 & 0.316 & $\star \star \star$ & 0.192 & $\star \star \star$ \\
\hline & STAT5A & 0.469 & $\star \star \star$ & 0.372 & $\star \star \star$ \\
\hline \multirow[t]{3}{*}{ Th9 } & TGFBR2 & 0.577 & 1 & 0.553 & $\star \star \star$ \\
\hline & IRF4 & 0.364 & $\star \star \star \star$ & 0.233 & $\star \star \star$ \\
\hline & SPI1 & 0.403 & 1 & 0.29 & $\star \star \star ~$ \\
\hline \multirow[t]{3}{*}{ Th17 } & IL-21R & 0.32 & $\star \star \star *$ & 0.149 & $\star \star \star *$ \\
\hline & IL-23R & 0.241 & $\star \star \star *$ & 0.198 & $\star \star \star *$ \\
\hline & STAT3 & 0.235 & $\star \star \star$ & 0.258 & 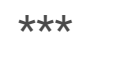 \\
\hline
\end{tabular}




\begin{tabular}{|c|c|c|c|c|c|}
\hline \multirow{2}{*}{$\begin{array}{l}\text { Cell type } \\
\text { Th22 }\end{array}$} & \multirow{2}{*}{$\begin{array}{l}\text { Gene marker } \\
\text { CCR10 }\end{array}$} & \multicolumn{2}{|l|}{ None } & \multicolumn{2}{|l|}{ Purity } \\
\hline & & 0.071 & 0.11 & 0.056 & 0.214 \\
\hline & AHR & 0.334 & $\star \star \star *$ & 0.318 & $\star \star \star \star$ \\
\hline \multirow[t]{3}{*}{ Treg } & FOXP3 & 0.317 & $\star \star \star$ & 0.17 & $\star \star \star$ \\
\hline & CCR8 & 0.324 & $\star \star \star$ & 0.201 & $\star \star \star$ \\
\hline & IL2RA & 0.196 & $\star \star \star$ & 0.055 & 0.221 \\
\hline \multirow[t]{3}{*}{$\mathrm{T}$ cell exhaustion } & PDCD1 & 0.142 & ** & -0.038 & 0.403 \\
\hline & CTLA4 & 0.228 & $\star \star \star$ & 0.048 & 0.283 \\
\hline & HAVCR2 & 0.309 & $\star \star \star *$ & 0.169 & $\star \star \star *$ \\
\hline \multirow[t]{2}{*}{ Macrophage } & CD68 & 0.331 & $\star \star \star \star$ & 0.232 & $\star \star \star *$ \\
\hline & ITGAM & 0.4 & 1 & 0307 & $\star \star \star \star$ \\
\hline \multirow[t]{4}{*}{ M1 } & NOS2 & 0.211 & $\star \star \star \star$ & 0.151 & $\star \star \star \star$ \\
\hline & ROS1 & 0.48 & 1 & 0.448 & 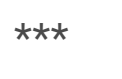 \\
\hline & IRF5 & 0.227 & $\star \star \star \star$ & 0.126 & ** \\
\hline & PTGS2 & 0.132 & $\star \star$ & 0.142 & $\star *$ \\
\hline \multirow[t]{3}{*}{ M2 } & ARG1 & 0.148 & $\star \star \star \star$ & 0.158 & $\star \star \star \star$ \\
\hline & CD163 & 0.325 & $\star \star \star \star$ & 0.216 & $\star \star \star \star ~$ \\
\hline & MRC1 & 0.49 & 1 & 0.429 & 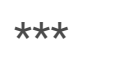 \\
\hline \multirow[t]{4}{*}{ TAM } & CCL2 & 0.299 & $\star \star \star \star$ & 0.191 & $\star \star \star \star$ \\
\hline & CCR5 & 0.338 & $\star \star \star \star$ & 0.183 & $\star \star \star \star$ \\
\hline & CD80 & 0.356 & $\star \star \star *$ & 0.226 & $\star \star \star \star$ \\
\hline & CD86 & 0.341 & $\star \star \star *$ & 0.211 & $\star \star \star *$ \\
\hline \multirow[t]{4}{*}{ Monocyte } & CD14 & 0.26 & $\star \star \star *$ & 0.134 & $\star \star$ \\
\hline & FCGR3A & 0.214 & $\star \star \star \star$ & 0.088 & 0.051 \\
\hline & FCGR3B & 0.185 & $\star \star \star *$ & 0.126 & $\star \star$ \\
\hline & CSF1R & 0.434 & $\star \star \star$ & 0.331 & 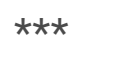 \\
\hline \multirow[t]{3}{*}{ NK } & XCL1 & -0.006 & 0.901 & -0.083 & 0.066 \\
\hline & KIR3DL1 & 0.032 & 0.468 & -0.045 & 0.314 \\
\hline & CD7 & 0.092 & * & -0.067 & 0.136 \\
\hline
\end{tabular}




\begin{tabular}{|c|c|c|c|c|c|}
\hline Cell type & Gene marker & None & & Purity & \\
\hline \multirow[t]{4}{*}{ Neutrophil } & FUT4 & 0.176 & $\star \star \star *$ & 0.135 & ** \\
\hline & MPO & 0.312 & $\star \star \star$ & 0.238 & $\star \star \star *$ \\
\hline & CEACAM8 & 0.386 & $\star \star \star$ & 0.403 & $\star \star \star *$ \\
\hline & ITGAM & 0.4 & 1 & 0.307 & $\star \star \star *$ \\
\hline \multirow[t]{3}{*}{ DC } & CD1C & 0.551 & $\star \star \star *$ & 0.505 & $\star \star \star *$ \\
\hline & THBD & 0.452 & $\star \star \star$ & 0.408 & $\star \star \star \star$ \\
\hline & ITGAX & 0.361 & $\star \star \star$ & 0.237 & $\star \star \star \star ~$ \\
\hline
\end{tabular}

Table 4

Correlation analysis between MFAP4 and macrophage associated markers in GEPIA

\begin{tabular}{|c|c|c|c|c|c|}
\hline \multirow[t]{2}{*}{ Cell type } & \multirow[t]{2}{*}{ Gene marker } & \multicolumn{2}{|l|}{ Tumor } & \multicolumn{2}{|c|}{ Normal } \\
\hline & & $\mathbf{R}$ & $\mathbf{p}$ & $\mathbf{R}$ & p \\
\hline \multirow[t]{4}{*}{ M1 } & NOS2 & 0.23 & $\star \star *$ & 0.67 & $\star \star *$ \\
\hline & ROS & 0.48 & 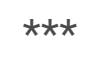 & 0.13 & 0.32 \\
\hline & IRF5 & 0.25 & $\star * *$ & -0.18 & 0.17 \\
\hline & PTGS2 & 0.13 & $\star \star$ & 0.23 & 0.077 \\
\hline \multirow[t]{3}{*}{ M2 } & ARG1 & 0.16 & $\star \star \star *$ & 0.026 & 0.85 \\
\hline & CD163 & 0.29 & $\star \star \star \star$ & -0.21 & 0.11 \\
\hline & MRC1 & 0.52 & $\star * *$ & 0.08 & 0.55 \\
\hline \multirow[t]{4}{*}{ TAM } & CCL2 & 0.31 & $\star \star *$ & 0.18 & 0.17 \\
\hline & CCR5 & 0.34 & $\star \star \star *$ & 0023 & 0.86 \\
\hline & CD80 & 0.35 & 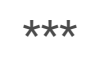 & 0.093 & 0.49 \\
\hline & CD86 & 0.36 & $\star * \star$ & -0.25 & 0.054 \\
\hline \multirow[t]{4}{*}{ Monocyte } & CD14 & 0.28 & 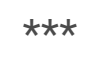 & 0.2 & 0.12 \\
\hline & FCGR3A & 0.24 & 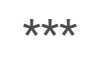 & -0.18 & 0.16 \\
\hline & FCGR3B & 0.17 & $\star * *$ & 0.22 & 0.089 \\
\hline & CSF1R & 0.46 & $\star \star \star *$ & 0.12 & 0.36 \\
\hline
\end{tabular}


In the present study, we conducted bioinformatics analysis on the publicly available RNA-sequencing data from different database, and the results showed that MFAP4 expression was downregulated in LUAD tissues compared to that in normal tissues at the protein and gene levels, thus indicating that MFAP4 could be a novel prognostic biomarker for LUAD. Our results showed that low-MFAP4 expression was associated with poor prognosis, and the possible underlying mechanism may be related to the immunosuppressive role of MFAP4 by altering the polarization of macrophages to increase the infiltration level of M2 macrophage.

The ECM, comprising fibrous proteins, proteoglycans, and matricellular-associated proteins, is a key component of the $\mathrm{TME}^{13}$. The differential organization and composition of the ECM components or the changes made by post-translational modifications affect the property of the ECM; for example, the stiffness of the stroma ${ }^{14}$. However, the stiffness of the ECM affects the cell recruited by other various matrix components, thereby influencing the physiological and pathological functions of cells and regulating tissue phenotype development and homeostasis ${ }^{15}$. One characteristic of tumor is abnormal levels of ECM-related protein that change the stiffness of tissue stroma ${ }^{16}$. Thus, as an ECM glycoproteinencoding gene, MFAP4 is a tumor-related gene. Moreover, several researchers have investigated the association between MFAP4 and different cancer types. Yang et al. ${ }^{17}$ found that high-MFAP4 expression correlated with improved OS in breast cancer. By contrast, Zhao et al. ${ }^{18}$ found that high-MFAP4 expression adversely affected the survival of neuroblastoma patients and played a role in regulating neuroblastoma cell differentiation. Furthermore, researchers have investigated MFAP4 as the downstream target of miRNA in LUAD. Feng et al. ${ }^{19}$ found that MFAP4, the putative target gene of miR147b, expression was downregulated in LUAD tissues, consistent with the result of this study, and associated with cell proliferation, invasion, and migration. A study ${ }^{20}$ showed that MFAP4 was the downstream target of a competitive endogenous RNA, mediated by C8orf34-as1, and that miR-671-5p played a role in LUAD cell migration and stemness. These studies collectively indicated that MFAP4 plays a crucial role in LUAD. However, these studies did not investigate the expression levels of MFAP4 and did not analyze the clinical survival data; hence, the prognostic value of MFAP4 in LUAD needs to be elucidated urgently. Here, we analyzed the MFAP4 expression in LUAD tissues at the mRNA level using the data from TCGA and GTEx database and at the protein level using data from HPA database. The results showed that MFAP4 was downregulated in LUAD tissues and associated with a worse prognosis, thus indicating the fact that MFAP4 could be a novel prognostic biomarker for LUAD.

Immune cell infiltrates ${ }^{21}$ and $\mathrm{ECM}^{22}$ are involved in TME and are crucial for the tumor progression; hence, it is important meaningful to elucidate whether there is an association between MFAP4 expression and immune cell infiltration. In this study, the immune infiltrates analysis revealed the most significant correlation between MFAP4 expression and macrophages infiltration; moreover, further analysis for macrophage subsets uncovered established that MFAP4 expression was positively correlated with M2 macrophage infiltration and negatively correlated with M1 macrophage infiltration; therefore, we hypothesize believed that MFAP4 plays a major role in macrophage polarization and thereby functionally more proximal to the M2 phenotypes. Generally, tumor-associated macrophages(TAMs) ,which 
differentiate from peripheral monocytes, are some of the most abundant population of immune cells in $\mathrm{TME}^{23}$, and they are divided into two major subtypes: classically activated macrophages (M1) and alternatively activated macrophages (M2) ${ }^{24}$. M1 phenotype is usually considered as "good" macrophages because of its anti-tumoral characteristics., They induce the production of proinflammatory factors, such as IL-12 and, TNFa, and are associated with chronic inflammation ${ }^{25}$. By contrast, M2 macrophages induce the production of anti-inflammatory cytokines, such as IL10 and ,TGF$\beta$, to promote tumor development, and hence, so they are considered as "bad" macrophages ${ }^{26}$. Moreover, IL10 are TGF- $\beta$ are immunosuppressive cytokines and curb the function of Th1 and Th2 cells, thereby modulating $T$ cell functions, and they are significantly overexpressed in human and mouse cancers ${ }^{27}$. Cortez et al. ${ }^{28}$ reported that the therapy with BMP7 regulates pro-inflammatory responses in the TAMs by decreasing the number of M2 macrophages and enhancing the tumor sensitivity to immunotherapy. In anaplastic thyroid carcinoma, Schürch et al. ${ }^{29}$ found that the patient survival was associated with the numbers of M2 macrophages and that the patients with prolonged survival showed reduced numbers of M2 macrophages. Interestingly, Kang et al. ${ }^{30}$ indicated that the accumulation of TAMs is associated with poor prognosis in colorectal cancer. These results indicate that MFAP4 may promote macrophage polarization towards an M2 phenotype and provide an immunosuppressive microenvironment for LUAD growth.

Our research has some limitations. First, this research is a bioinformatics analysis based on the public database. Although the results were obtained from analyzing different datasets, further experimental research is needed to validate and shed light on the potential mechanisms. Second, considering the data in TCGA and GEO were not updated timely and the provided clinical data was not sufficient, further large clinical studies with large sample sizes and long follow-up duration are needed. Additionally, the negative associations with tumor purity indicated that MFAP4 is highly expressed in cells in the TME, and singlecell analysis needs to be performed to interpret the reliability of these result.

In summary, we found that MFAP4 expression was downregulated in LUAD tissues at the gene and protein levels. Low expression levels of MFAP4 were associated with adverse survival and could be a potential prognostic biomarker for LUAD. Moreover, we also showed that MFAP4 expression was correlated with immune cell infiltration, especially with macrophages infiltration, and might play a vital role in macrophage polarization by promoting the M2 phenotype numbers.

\section{Materials And Methods}

\section{Data collection and processing}

The TPM-normalized RNA-sequencing data from the GTEx and TCGA database (https://cancergenome.nih.gov/), which were processed through TOIL, were downloaded from the UCSC Xena database (https://xenabrowser.net/datapages/). These data included the information of 347 healthy tissues and 515 cancerous tissues. The expression levels of MFAP4 in LUAD and healthy tissues 
were presented as scatter plots. The HPA database (https://www.proteinatlas.org/) is an online database that indicates the difference in expression levels between normal and tumor tissues at the protein level. In this study, we investigated the protein expression of MFAP4 in LUAD and normal tissues using HPA.

\section{Survival analysis}

We used the R survival package to analyze the survival of patients with MFAP4 data from TCGA database and supplementary data ${ }^{31}$, furthermore, PrognoScan database(http://www.prognoscan.org/) 32 was used to establish the prognostic value of MFAP4 in LUAD. PrognoScan database provides a powerful platform for assessing the biological relationship between potential tumor markers and prognosis. Two GEO datasets (GSE13213 and GSE31210) were applied, and Cox proportional hazards regression models were used to estimate the hazard ratios.

\section{Immune infiltration analysis}

The relationship between the MFAP4 expression and immune infiltration was analyzed using the TIMER and GEPIA databases. TIMER is a comprehensive online tool used to estimate the association of tumor gene expression data and immune cell infiltration (http://cistrome.org/TIMER/) ${ }^{33}$. It was used to analyze the data from TCGA to determine the abundance of six TIIC subsets (B cells, CD $4^{+} \mathrm{T}$ cells, CD $8^{+} \mathrm{T}$ cells, macrophages, neutrophils, and dendritic cells). GEPIA (http://gepia.cancer-pku.cn/) $)^{34}$ is a web-based tool that provides key interactive functions based on the data from TCGA and the GTEx databases. Thus, we explored the association between TIICs and MFAP4 expression in LUAD tissues using TIMER and GEPIA. Moreover, the expression of MFAP4 in pan-cancer tissues was also analyzed using TIMER.

\section{Statistical analyze}

All data in this study were analyzed using the R software (version 3.6.3). The expression of MFAP4 in pan-cancer was analyzed using the TIMER database. For the data obtained from TCGA and GTEx database, Shapiro-Wilk normality test was used for data normalization and Wilcoxon rank-sum test or paired t-test were performed as needed. The prognostic value of MFAP4 expression in LUAD tissues was determined using Kaplan-Maier survival and Cox regression analyses with the survival package (version 3.2-10). We visualized the results of the Cox regression univariate analysis with forest plots; moreover, a nomogram was drawn based on the results of multivariate analysis and evaluated using the calibration curve. Furthermore, data visualization was performed with the survminer (version 0.4.9), ggplot2 package, or rms package. A P-value $<0.05$ indicated statistical difference.

\section{Declarations}

\section{Data Availability}

The data that support the findings of this study are available from the Cancer Genome Atlas (TCGA, http://cancergenome.nih.gov/) and Gene Expression Omnibus (GEO, https://www.ncbi.nlm.nih.gov/ geo/), accession number GSE13213 and GSE31210. 


\section{Ethics declarations}

All human clinical data in this study were obtained from publically accessible databases and were in accordance with the ethical standards of the institutional and/or national research committee and with the 1964 Helsinki declaration and its later amendments or comparable ethical standards.

\section{Acknowledgements}

Funding: This study was supported by Project of Guangdong Medical Science and Technology Research Funding(C2019015).

Abbreviations: Microfiber-associated protein 4: MFAP4; lung adenocarcinoma: LUAD; extracellular matrix: ECM; The Cancer Genome Atlas :TCGA; Genotype-Tissue Expression: GTEx; Gene Expression Omnibus: GEO; the tumor microenvironment :TME; the Human Protein Atlas :HPA; CR: complete response; PD :progressive disease; SD :stable disease; PR :partial response; Tfh :Follicular helper T cell; Th :T helper cell; Treg :Regulatory T cell; TAM :Tumor-associated macrophage; NK :natural killer cell; DC :dendritic cell; None :Correlation without adjustment; Purity :Correlation adjusted by purity; Cor :R value of Spearman's correlation.

\section{Author Contributions}

YJ, ZYL and WFY conceived and designed the research, WFY and ZYL wrote the manuscript, TGY and YHL collected and interpreted the data, YGC revised the paper. All authors read and approved the manuscript.

\section{Additional Information}

Competing Interests: The authors declare no competing interests.

\section{References}

1. Bray, F. et al. Global cancer statistics 2018: GLOBOCAN estimates of incidence and mortality worldwide for 36 cancers in 185 countries. CA Cancer J Clin, 68, 394-424 https://doi.org/10.3322/caac.21492 (2018).

2. Hua, X. et al. Genetic and epigenetic intratumor heterogeneity impacts prognosis of lung adenocarcinoma. Nat Commun, 11, 2459 https://doi.org/10.1038/s41467-020-16295-5 (2020).

3. Herbst, R. S. et al. Selective Oral Epidermal Growth Factor Receptor Tyrosine Kinase Inhibitor ZD1839 Is Generally Well-Tolerated and Has Activity in Non-Small-Cell Lung Cancer and Other Solid Tumors: Results of a Phase I Trial. Journal of Clinical Oncology, 20, 3815-3825 https://doi.org/10.1200/jco.2002.03.038 (2002).

4. Mok, T. et al. Updated overall survival and final progression-free survival data for patients with treatment-naive advanced ALK-positive non-small-cell lung cancer in the ALEX study. Ann Oncol, 31, 
1056-1064 https://doi.org/10.1016/j.annonc.2020.04.478 (2020).

5. Boumahdi, S. \& de Sauvage, F. J. The great escape: tumour cell plasticity in resistance to targeted therapy. Nat Rev Drug Discov, 19, 39-56 https://doi.org/10.1038/s41573-019-0044-1 (2020).

6. Scagliotti, G. V. et al. Phase III study comparing cisplatin plus gemcitabine with cisplatin plus pemetrexed in chemotherapy-naive patients with advanced-stage non-small-cell lung cancer. $J$ Clin Oncol, 26, 3543-3551 https://doi.org/10.1200/JC0.2007.15.0375 (2008).

7. Mecham, R. P. \& Gibson, M. A. The microfibril-associated glycoproteins (MAGPs) and the microfibrillar niche. Matrix Biol, 47, 13-33 https://doi.org/10.1016/j.matbio.2015.05.003 (2015).

8. Molleken, C. et al. Detection of novel biomarkers of liver cirrhosis by proteomic analysis., 49, 12571266 https://doi.org/10.1002/hep.22764 (2009).

9. Johansson, S. L. et al. Microfibrillar-associated protein 4: a potential biomarker of chronic obstructive pulmonary disease. Respir Med, 108, 1336-1344 https://doi.org/10.1016/j.rmed.2014.06.003 (2014).

10. Wulf-Johansson, H. et al. Localization of microfibrillar-associated protein 4 (MFAP4) in human tissues: clinical evaluation of serum MFAP4 and its association with various cardiovascular conditions. PloS one, 8, e82243 https://doi.org/10.1371/journal.pone.0082243 (2013).

11. Wang, J. K. et al. MFAP2 promotes epithelial-mesenchymal transition in gastric cancer cells by activating TGF-beta/SMAD2/3 signaling pathway. Onco Targets Ther, 11, 4001-4017 https://doi.org/10.2147/OTT.S160831 (2018).

12. Yang, X. et al. MFAP5 and TNNC1 Potential markers for predicting occult cervical lymphatic metastasis and prognosis in early stage tongue cancer. (2017).

13. Mouw, J. K., Ou, G. \& Weaver, V. M. Extracellular matrix assembly: a multiscale deconstruction. Nature reviews. Molecular cell biology, 15, 771-785 https://doi.org/10.1038/nrm3902 (2014).

14. Pickup, M. W., Mouw, J. K. \& Weaver, V. M. The extracellular matrix modulates the hallmarks of cancer. EMBO Rep, 15, 1243-1253 https://doi.org/10.15252/embr.201439246 (2014).

15. Kai, F., Drain, A. P. \& Weaver, V. M. The Extracellular Matrix Modulates the Metastatic Journey. Dev Cell, 49, 332-346 https://doi.org/10.1016/j.devcel.2019.03.026 (2019).

16. Charras, G. \& Sahai, E. Physical influences of the extracellular environment on cell migration. Nature reviews. Molecular cell biology, 15, 813-824 https://doi.org/10.1038/nrm3897 (2014).

17. Yang, J. et al. Integrated analysis of microfibrillar-associated proteins reveals MFAP4 as a novel biomarker in human cancers. (2018).

18. Zhao, Z. et al. microRNA-449a functions as a tumor suppressor in neuroblastoma through inducing cell differentiation and cell cycle arrest. RNA biology, 12, 538-554 https://doi.org/10.1080/15476286.2015.1023495 (2015).

19. Feng, Y. Y. et al. MicroRNA-147b promotes lung adenocarcinoma cell aggressiveness through negatively regulating microfibril-associated glycoprotein 4 (MFAP4) and affects prognosis of lung adenocarcinoma patients., 730, 144316 https://doi.org/10.1016/j.gene.2019.144316 (2020). 
20. Han, P. et al. Identification of a Novel Cancer Stemness-Associated ceRNA Axis in Lung Adenocarcinoma via Stemness Indices Analysis. Oncology Research Featuring Preclinical and Clinical Cancer Therapeutics, 28, 715-729 https://doi.org/10.3727/096504020x16037124605559 (2021).

21. Coussens, L. M., Zitvogel, L. \& Palucka, A. K. Neutralizing tumor-promoting chronic inflammation: a magic bullet? Science (New York, N.Y.), 339, 286-291 https://doi.org/10.1126/science.1232227 (2013).

22. Oudin, M. J. et al. Tumor Cell-Driven Extracellular Matrix Remodeling Drives Haptotaxis during Metastatic Progression. Cancer Discov, 6, 516-531 https://doi.org/10.1158/2159-8290.CD-15-1183 (2016).

23. Noy, R. \& Pollard, J. W. Tumor-associated macrophages: from mechanisms to therapy., 41, 49-61 https://doi.org/10.1016/j.immuni.2014.06.010 (2014).

24. Dehne, N., Mora, J., Namgaladze, D., Weigert, A. \& Brune, B. Cancer cell and macrophage cross-talk in the tumor microenvironment. Curr Opin Pharmacol, 35, 12-19

https://doi.org/10.1016/j.coph.2017.04.007 (2017).

25. Jeannin, P., Paolini, L., Adam, C. \& Delneste, Y. The roles of CSFs on the functional polarization of tumor-associated macrophages. FEBS J, 285, 680-699 https://doi.org/10.1111/febs.14343 (2018).

26. Sica, A. et al. Macrophage polarization in tumour progression. Semin Cancer Biol, 18, 349-355 https://doi.org/10.1016/j.semcancer.2008.03.004 (2008).

27. Kim, O. H. et al. Proangiogenic TIE2(+)/CD31 (+) macrophages are the predominant population of tumor-associated macrophages infiltrating metastatic lymph nodes. Mol Cells, 36, 432-438 https://doi.org/10.1007/s10059-013-0194-7 (2013).

28. Cortez, M. A. et al. Bone morphogenetic protein 7 promotes resistance to immunotherapy. Nat Commun, 11, 4840 https://doi.org/10.1038/s41467-020-18617-z (2020).

29. Schurch, C. M. et al. Targeting CD47 in Anaplastic Thyroid Carcinoma Enhances Tumor Phagocytosis by Macrophages and Is a Promising Therapeutic Strategy. Thyroid, 29, 979-992 https://doi.org/10.1089/thy.2018.0555 (2019).

30. Chen, J. J. et al. Tumor-associated macrophages: the double-edged sword in cancer progression. $J$ Clin Oncol, 23, 953-964 https://doi.org/10.1200/JC0.2005.12.172 (2005).

31. Liu, J. et al. An Integrated TCGA Pan-Cancer Clinical Data Resource to Drive High-Quality Survival Outcome Analytics. Cell 173, 400-416 e411, doi:10.1016/j.cell.2018.02.052 (2018).

32. Mizuno, H., Kitada, K., Nakai, K. \& Sarai, A. PrognoScan: a new database for meta-analysis of the prognostic value of genes. BMC Med Genomics, 2, 18 https://doi.org/10.1186/1755-8794-2-18 (2009).

33. Li, T. et al. TIMER: A Web Server for Comprehensive Analysis of Tumor-Infiltrating Immune Cells. Cancer Res, 77, e108-e110 https://doi.org/10.1158/0008-5472.can-17-0307 (2017).

34. Tang, Z. et al. GEPIA: a web server for cancer and normal gene expression profiling and interactive analyses. Nucleic Acids Res, 45, W98-W102 https://doi.org/10.1093/nar/gkx247 (2017). 
Figures

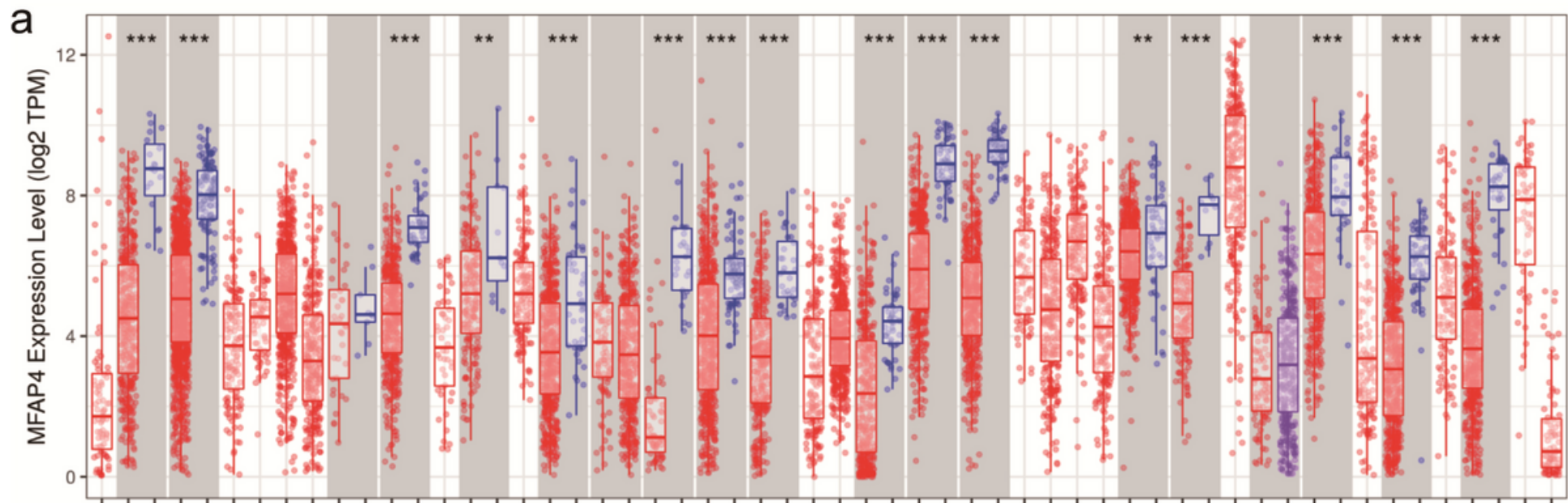

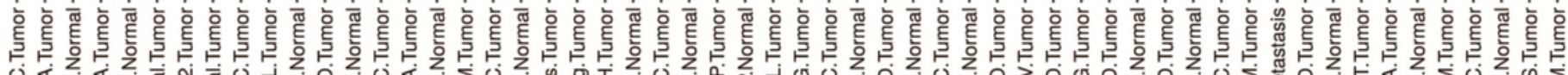

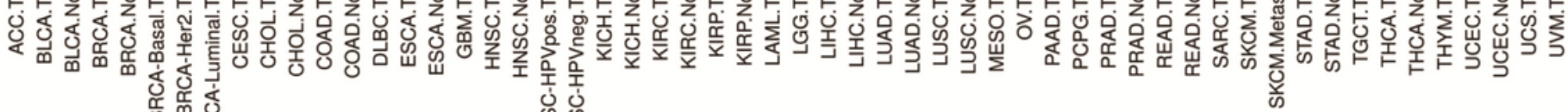

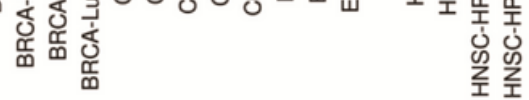
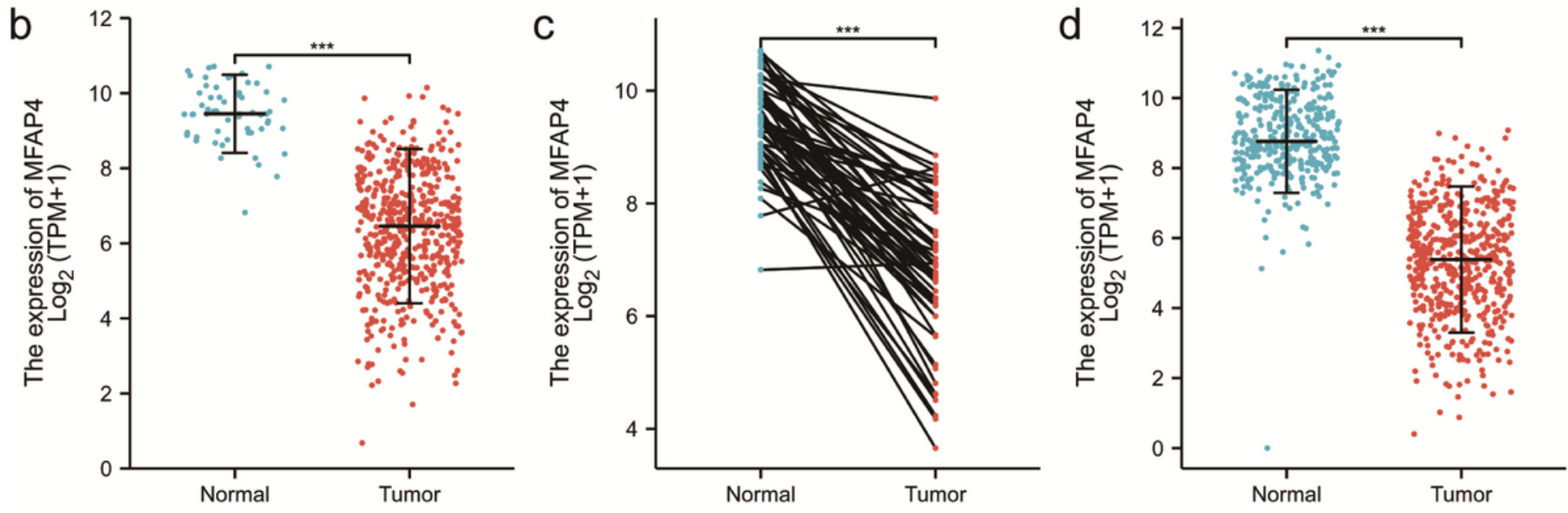

Figure 1

Down-regulated expression of MFAP4 in Pan-cancer and LUAD.(A)MAFP4 expression in pan-cancer data from TCGA. (B-C)MFAP4 expression in normal and tumor tissues in LUAD from TCGA database.

(D)MFAP4 expression in normal and tumor tissues in LUAD from TCGA and GTEx database.

${ }^{\star} p<0.05,{ }^{* *} p<0.01$, ${ }^{\star * *} p<0.001$ 
a

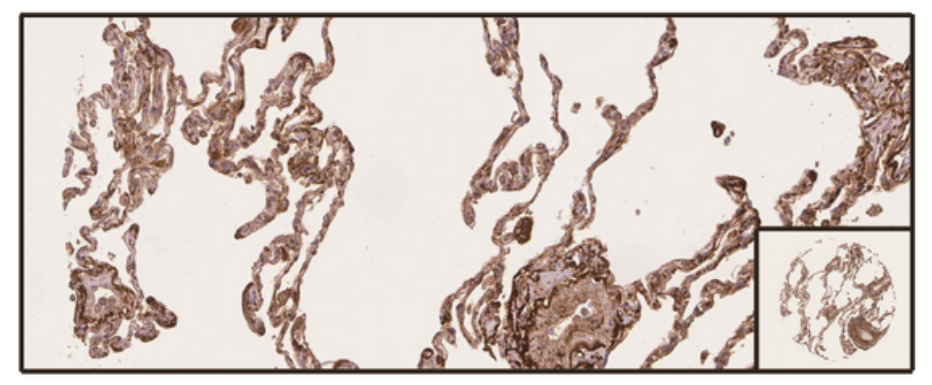

b

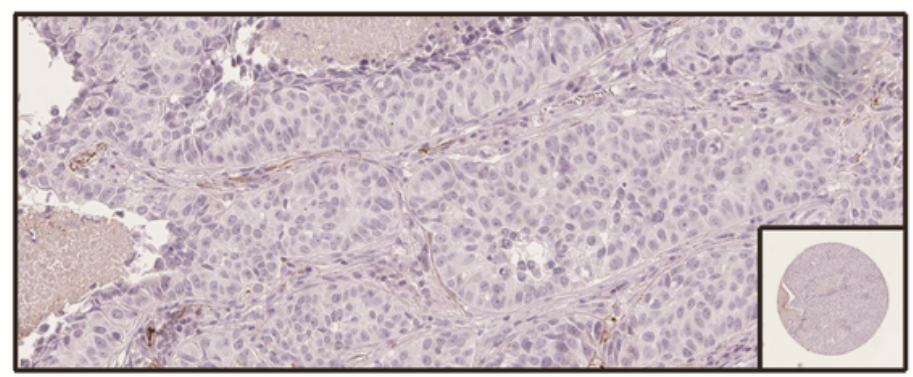

Figure 2

Low MFAP4 expression in LUAD on protein level.(A)normal tissue, (B)LUAD tissue.
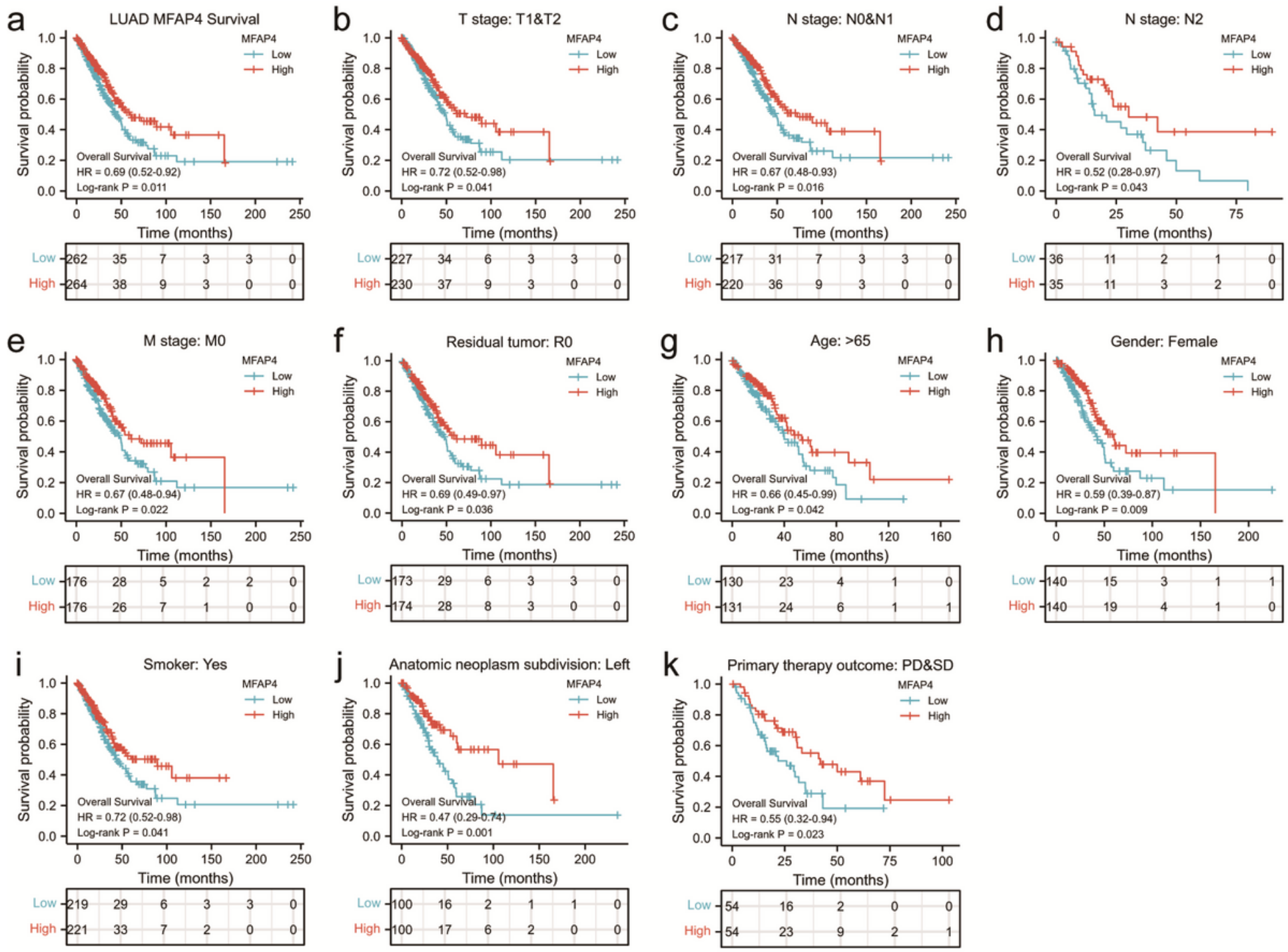

\section{Figure 3}

Low MFAP4 expression associated with poor prognosis in (A)LUAD patients, (B-K)subgroup Kaplan-Meier survival analysis for TNM stage, residual tumor, age, gender, smoker, anatomic neoplasm subdivision and primary therapy outcome. 
a

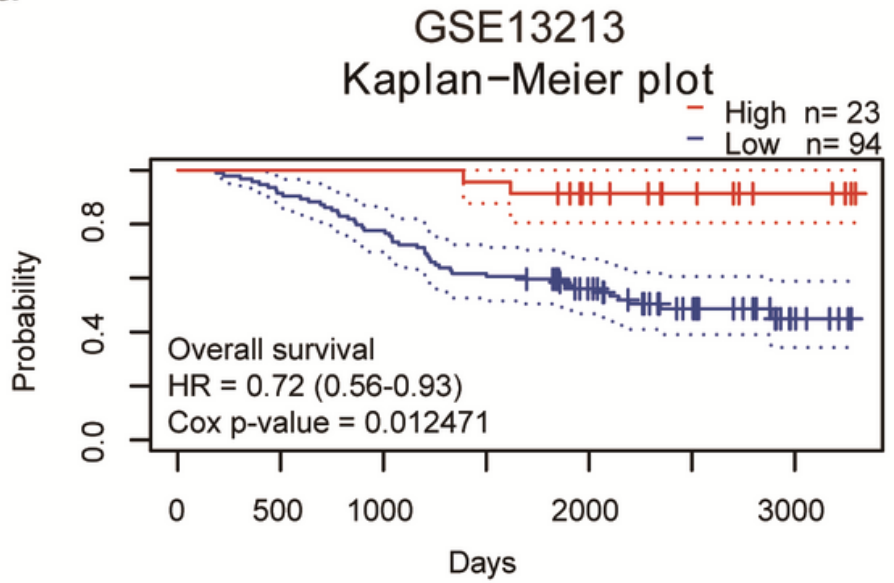

b

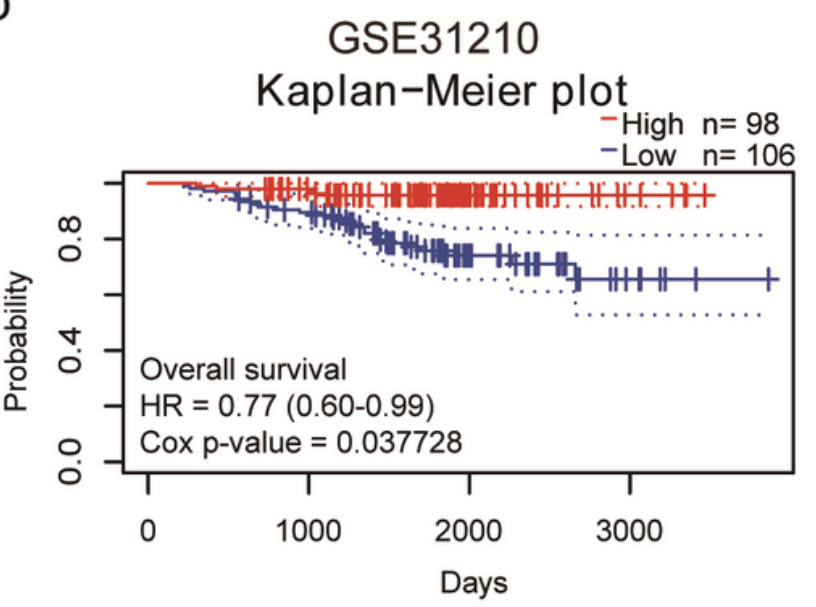

\section{Figure 4}

Low MFAP4 expression associated with poor prognosis in LUAD patients data from (A)GSE13213, (B)GSE31210.

\begin{tabular}{ccccc}
\hline Characteristics & Total(N) & HR(95\% Cl) & P value \\
\hline T stage (T3\&T4 vs. T1\&T2) & 523 & $2.317(1.591-3.375)$ & $<0.001$ \\
N stage (N1\&N2\&N3 vs. N0) & 510 & $2.601(1.944-3.480)$ & $<0.001$ \\
M stage (M1 vs. M0) & 377 & $2.136(1.248-3.653)$ & 0.006 \\
Pathologic stage (Stage III\&Stage IV vs. Stage I\&Stage II) & 518 & $2.664(1.960-3.621)$ & $<0.001$ \\
Primary therapy outcome (PR\&CR vs. PD\&SD) & 439 & $0.377(0.268-0.530)$ & $<0.001$ \\
Gender (Male vs. Female) & 526 & $1.070(0.803-1.426)$ & 0.642 \\
Age (>65 vs. <=65) & 516 & $1.223(0.916-1.635)$ & 0.172 \\
Residual tumor (R1\&R2 vs. R0) & 363 & $3.879(2.169-6.936)$ & 0.81 \\
Anatomic neoplasm subdivision (Right vs. Left) & 512 & $1.037(0.770-1.397)$ & 0.706 \\
Snatomic neoplasm subdivision2 (Peripheral Lung vs. Central Lung) & 182 & $0.913(0.570-1.463)$ & 1 & 0.591 \\
MFAP4 (Low vs. High) & 512 & $0.894(0.592-1.348)$ & 0.011 \\
\hline
\end{tabular}

b

Points

Pathologic stage

Residual tumor

Primary therapy outcome

MFAP4

Total Points

Linear Predictor

1-year Survival Probability

3-year Survival Probability

5-year Survival Probability

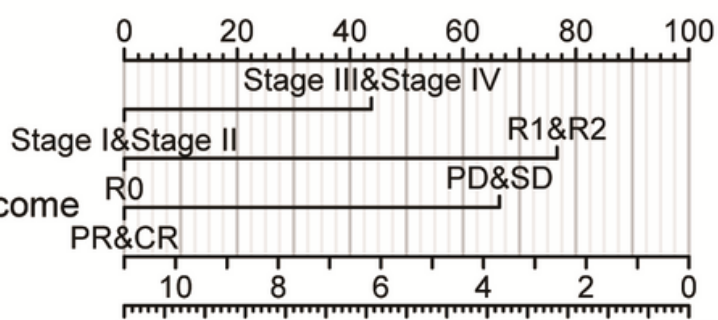

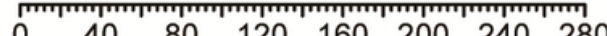

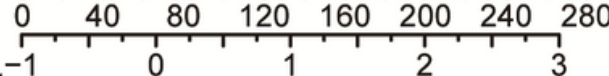

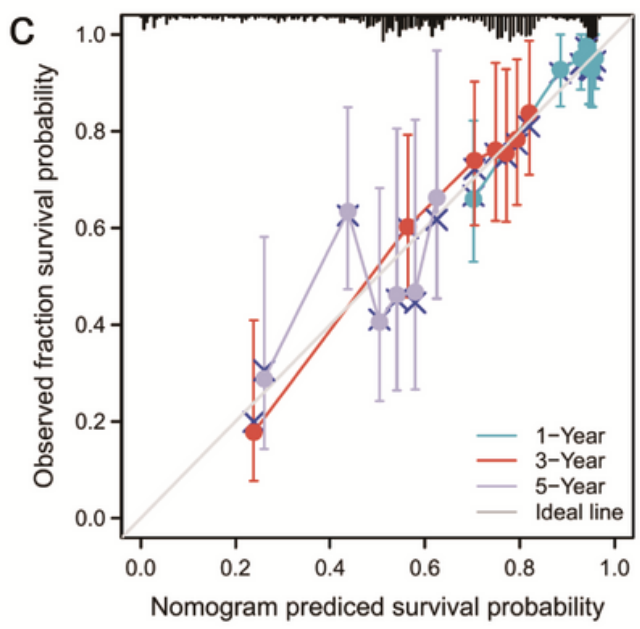

Figure 5 
Survival association of clinical characteristics in LUAD patients. (A)Forest plot of the prognostic value of chlinical risk factors, (B)nomogram to predict the OS at 1,3 and 5 years, (C)Calibration plots showed that predictive capability of 1 and 3 years was notably superior than 5 years.

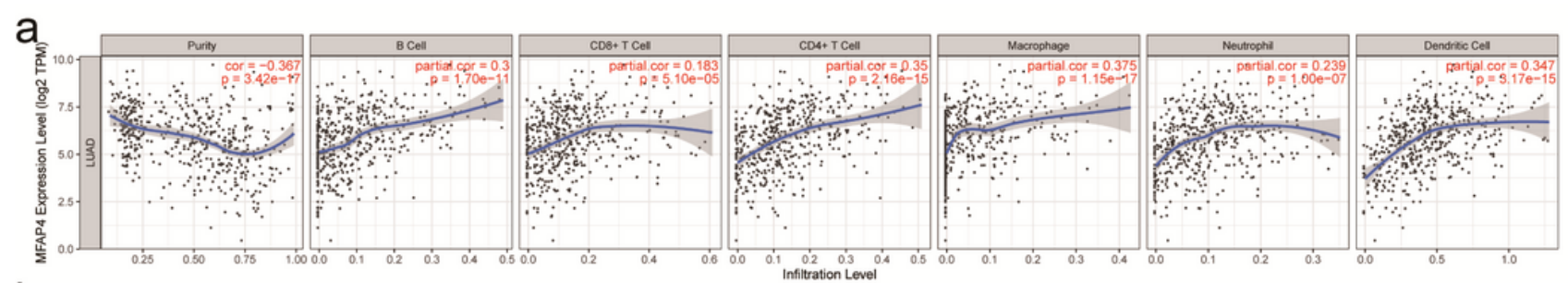

$\mathrm{b}$
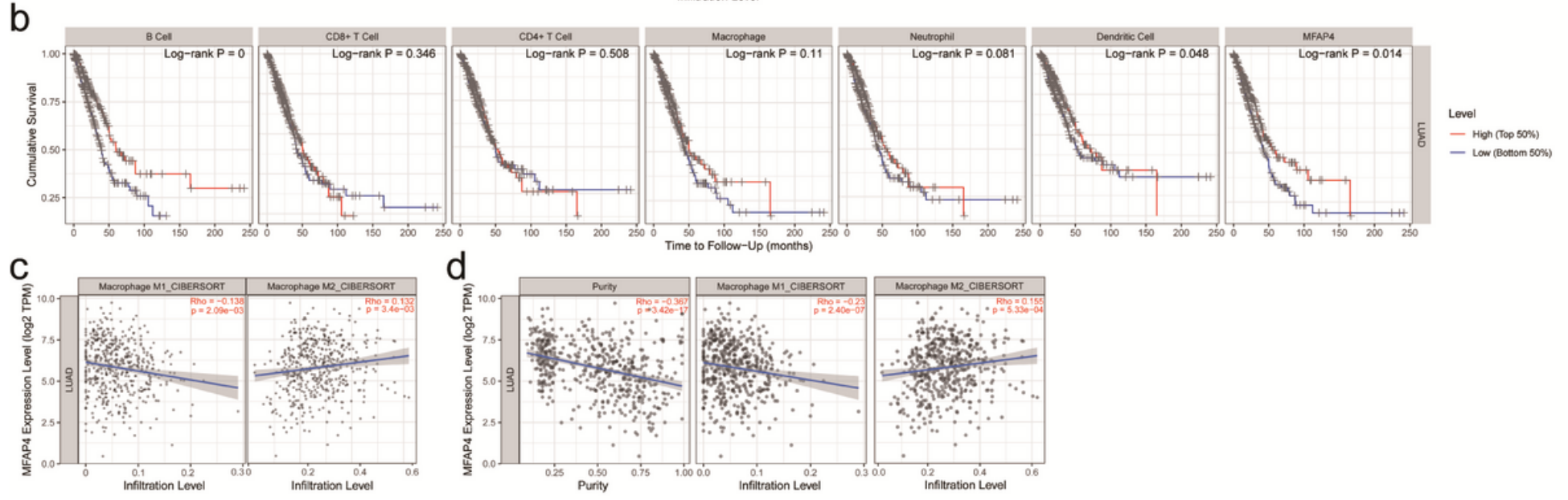

Figure 6

MFAP4 expression is correlated with immune cell infiltrations in LUAD.(A)MFAP4 expression is associated with six tumor-infiltrating immune cells subsets. (B)Kaplan-Meier plots of MFAP4 expression and the infiltration level of six tumor-infiltrating immune cells subsets. (C)MFAP4 expression and infiltration level of M1/M2 macrophages before purity adjustment. (D) MFAP4 expression and infiltration level of M1/M2 macrophages after purity adjustment.
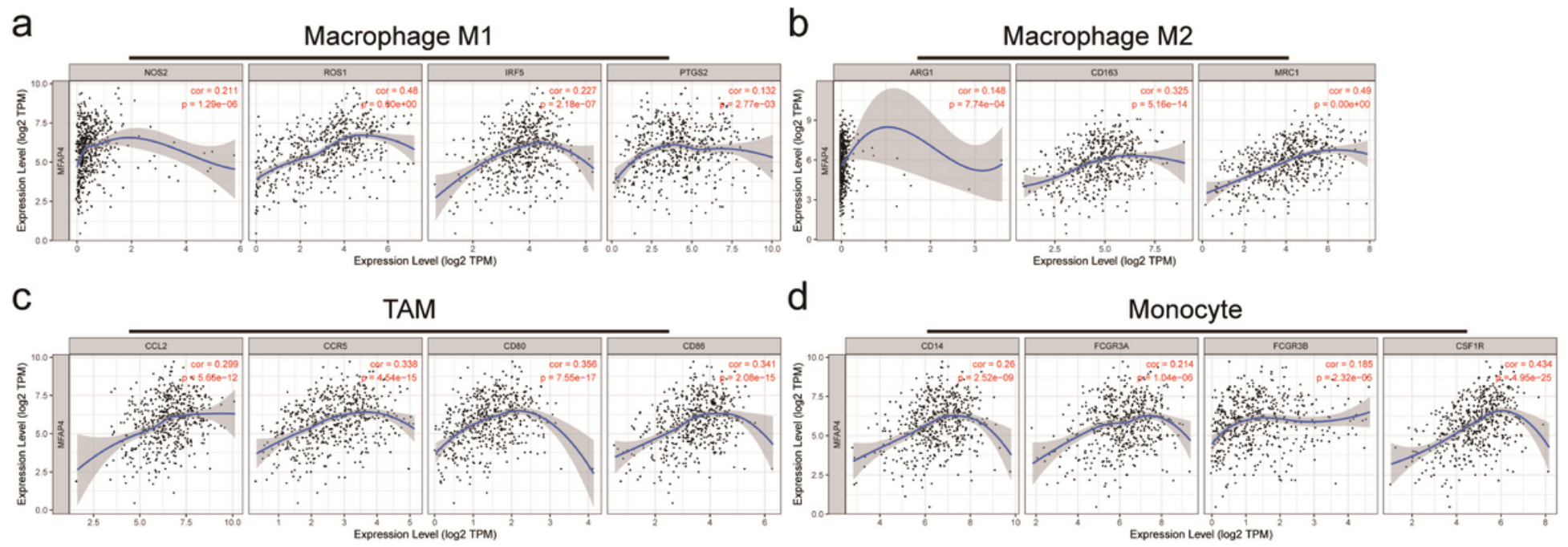

Figure 7 
MFAP4 expression correlates with monocyte and macrophage-related immune cell infiltration. (A)M1 macrophage phenotype markers, (B)M2 macrophage phenotype markers, (C)TAM macrophage phenotype markers, (D)Monocyte macrophage phenotype markers. 